\title{
Le Congrès général de Marseille 2013
}

Le $22^{\mathrm{e}}$ Congrès général de la Société Française de Physique, organisé par la section locale Provence de la SFP, avec le soutien de la section Côte d'Azur, s'est déroulé à Marseille, du $1^{\mathrm{er}}$ au 5 juillet 2013. Les participants ont été accueillis au site Saint-Charles de la Faculté des sciences de l'Université d'Aix-Marseille, situé en centre-ville. Le congrès bénéficiait du cadre de « Marseille-Provence 2013, capitale européenne de la culture».

\section{Quelques chiffres}

- 21 membres du Comité scientifique

- 31 membres du Comité d'organisation

- 16 conférences plénières (dont deux destinées au grand public)

- 17 minicolloques (dont deux tables rondes et un atelier)

- 14 entreprises exposantes

- 21 partenaires financeurs (voir liste p. 37)

- 600 inscrits (dont 110 doctorants et postdoctorants, 31 étrangers et 24 retraités)

- Budget : $200 \mathrm{k€}$
Le contenu scientifique du congrès a été élaboré par le comité scientifique, constitué essentiellement de représentants des divisions thématiques de la SFP. Comme pour les précédentes éditions, le programme était composé de conférences plénières et de minicolloques.

Nous avons essayé de construire un programme attractif qui couvre l'ensemble des domaines de la physique, des plus fondamentaux aux plus appliqués, et qui montre bien l'interdisciplinarité et les interactions très fortes de la physique avec les disciplines voisines comme la chimie, l'astrophysique, la biologie ou la médecine. Nous avons aussi essayé de montrer l'importance de notre discipline et l'intérêt des physiciens pour de nombreuses questions de société, par le minicolloque et la table ronde sur l'énergie qui a été un point fort $\mathrm{du}$ congrès, mais aussi par ceux sur les femmes et la physique (voir article, p. 38), les publications scientifiques, ou celui sur la communication et la culture scientifique.

\section{Les conférences plénières}

Le congrès s'est ouvert sur une conférence de Bernard Bigot, Administrateur Général du CEA, qui a présenté une revue sur la complémentarité entre l'énergie nucléaire et les énergies renouvelables qui permettront de réduire la consommation en énergies fossiles et carbonées afin de limiter les risques sanitaires, environnementaux et de changement climatique. Dans le domaine de l'énergie, un autre défi majeur des vingt prochaines années est, sans aucun doute, le développement de nouvelles technologies pour le stockage de l'énergie. Jean-Marie Tarascon, professeur au Collège de France et chimiste, nous a montré les potentialités des accumulateurs avancés à ions lithium et les nouvelles questions qu'ils posent pour les physiciens.

Un des évènements marquants de l'année a bien sûr été la découverte du boson de Higgs. À partir de cette découverte, Sergio Bertolucci, directeur scientifique du CERN, a fait le point sur le futur de

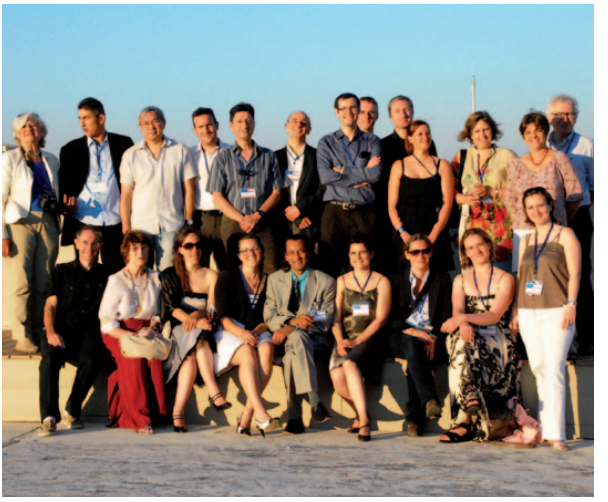

Le comité d'organisation du congrès

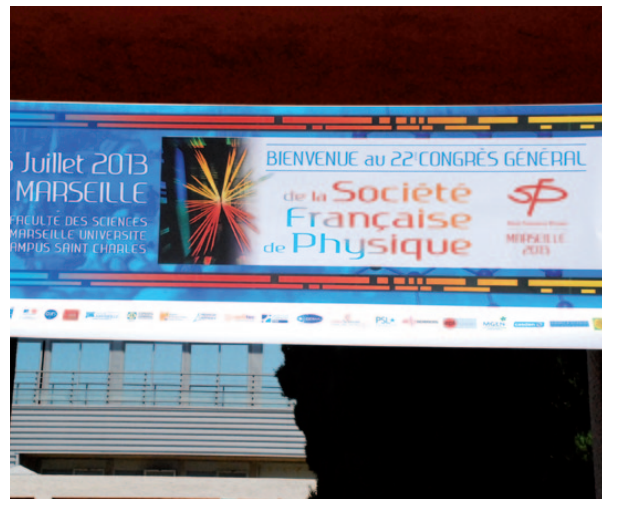

La banderole de bienvenue

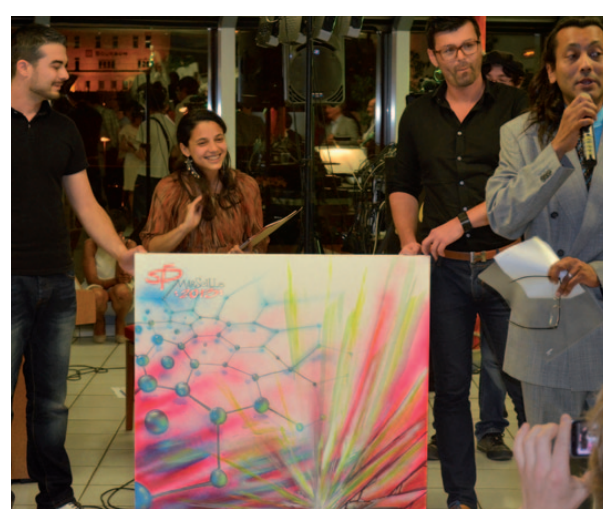

La lauréate du prix poster : Mlle Nahed Sihem Bounoua 


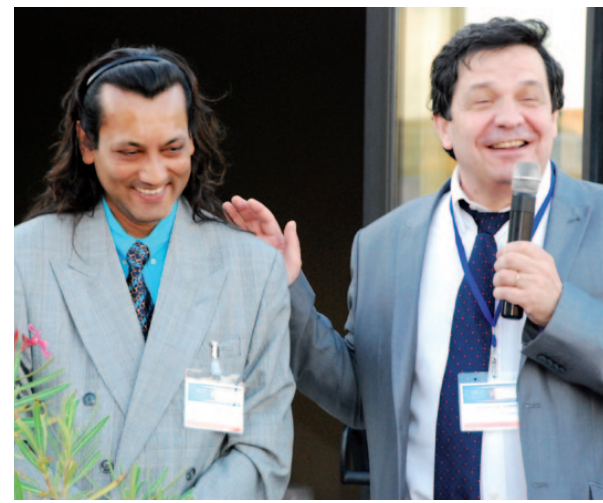

Dave Lollman (à gauche) et Jean-François Joanny (à droite).

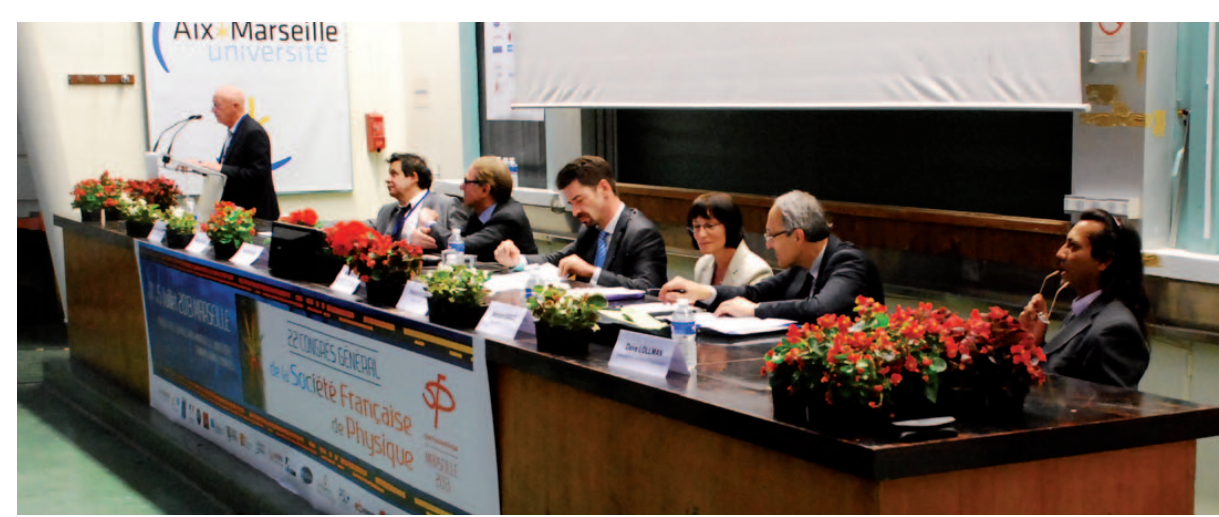

La cérémonie d'ouverture. De gauche à droite : M. Lannoo (président de la SFP), J.F. Joanny, Y. Berland (président de l'Université d'Aix-Marseille), M. Moroni (représentant G. Fioraso, MESR), P. Roubin (directrice-adjointe de I'INP/CNRS), B. Bigot et D. Lollman. l'accélérateur LHC et la recherche de nouvelles particules. La physique fondamentale a fait l'objet de plusieurs exposés de physique des particules ou d'astrophysique. Christophe Yèche nous a parlé de l'énergie noire et de l'accélération de l'expansion de l'Univers. Jean-Pierre Lasota a fait un exposé sur la détermination du spin des trous noirs et sur la modélisation des jets relativistes observés dans le cœur des galaxies et dont la source est la rotation des trous noirs. Enfin, Pierre Pizzochero nous a montré comment les propriétés physiques extrêmes des étoiles compactes (étoiles à neutrons et " étoiles étranges ") fournissent un outil exceptionnel pour étudier les propriétés de l'espace-temps, l'équation d'état des systèmes denses et la physique des interactions forte et faible.

Une part importante des exposés a été consacrée aux matériaux et à la physique de la matière condensée. Stefan Enoch nous a parlé des métamatériaux qui permettent d'obtenir des propriétés optiques très contre-intuitives comme l'invisibilité, et de l'application de ces concepts, à une échelle beaucoup plus grande, aux ondes sismiques. Wolfgang Wernsdorfer nous a introduit à l'électronique moléculaire, à la spintronique et en particulier à la manipulation d'aimants à l'échelle d'une molécule. Deux exposés ont abordé des aspects plus théoriques : Jan Zaanen a montré comment l'on pouvait utiliser des méthodes venant de la gravité quantique pour étudier des systèmes d'électrons fortement corrélés ; Peter Wölfle, qui est le lauréat 2013 du prix Gentner-Kastler, a discuté les propriétés critiques des métaux au voisinage d'une transition de phase quantique, avec des applications aux systèmes antiferromagnétiques et aux fermions lourds.
La physique des lasers a fait l'objet de deux exposés dans des domaines très différents. François Amiranoff a présenté la physique avec les nouveaux lasers multi-petawatt et leurs applications pour l'accélération de faisceaux d'électrons et d'ions bien au-delà du $\mathrm{GeV}$, ou la production de sources intenses femtoseconde et attoseconde de rayons $\mathrm{X}$ et de rayons gamma. Nathalie Picqué a décrit un nouveau mode de spectroscopie par peignes de fréquences femtosecondes et ses applications à la spectroscopie moléculaire à large bande.

Les deux derniers exposés portaient sur de nouvelles méthodes d'imagerie en géophysique et en biophysique. Barbara Romanowicz, a présenté les progrès récents dans l'imagerie du manteau terrestre en utilisant les ondes sismiques. Enfin, Mickael Tanter nous a montré les possibilités spectaculaires de l'échographie ultrarapide en illustrant son propos de nombreuses applications médicales.

Deux conférences " grand public » ont aussi été présentées le mercredi en fin d'après-midi. Ina Reiche a décrit les méthodes spectroscopiques qui peuvent donner des informations cachées sur le savoir-faire et les modes de vie des sociétés anciennes, en caractérisant la nature des matériaux du patrimoine culturel et leurs propriétés physico-chimiques. François Bouchet a présenté les premiers résultats cosmologiques de la mission Planck, rendus publics le 21 mars 2013. Il a décrit la carte $\mathrm{du}$ rayonnement cosmologique du fond du ciel, sa signification et son interprétation, et ce que l'on en a tiré sur les premiers instants du Big-Bang et sur l'évolution ultérieure de l'Univers.

\section{Les remises de prix}

\section{Les prix du concours " poster "}

Sur 102 posters affichés, un jury a sélectionné trois prix selon des critères de présentation, contenu et qualité visuelle. Ces prix, sponsorisés par EPL (Europhysics Letters), ont été attribués à :

- Nahed Sihem Bounoua, Laboratoire de Physique de la Matière Condensée, CNRS, Université de Nice SophiaAntipolis. Poster : "Viscosité de suspensions de fibres non-browniennes ".

- Louis-Charles Garnier, Institut des Nano-Sciences de Paris, CNRS, Université Paris 6. Poster : "Étude structurale et magnétique des phases $\mathrm{Fe}-\mathrm{N}$ formées par implantation d'ions azote dans une couche mince de fer" .

- Igor Andriyash, Laboratoire d'Optique Appliquée, CNRS, École Polytechnique, Palaiseau. Poster : "Betatron emission from accelerated electrons scattered in a high intensity optical lattice".

Le prix «lauréat " a été attribué à Nahed Sihem Bounoua, qui a également reçu un tableau d'art, confectionné en "live" pendant la soirée de gala, par Patrick Liveneau, artiste contemporain (voir photo).

\section{Le prix Gentner-Kastler 2013}

Ce prix, attribué conjointement par la DPG (Société allemande de physique) et la SFP, a été remis à Peter Wölfle (Institut de la matière condensée, Université de Karlsruhe), en reconnaissance de ses contributions exceptionnelles à la théorie des systèmes de la matière condensée (voir notice, p. 41). La cérémonie de remise de ce prix a eu lieu le mercredi 3 juillet, juste avant sa brillante conférence plénière. 


\section{$\gg\rangle$ \\ Les minicolloques}

Le congrès comportait dix-sept minicolloques, organisés en sessions parallèles.

1 - Le minicolloque Énergie s'est ouvert sur une table ronde qui a fait le point sur les grands enjeux scientifiques et technologiques. Des sessions ont été consacrées aux énergies nucléaires de fission et de fusion, à l'utilisation des photons, au transport et à la conversion, et aux matériaux. Les orateurs ont fait un effort tout à fait exceptionnel pour rendre clairs les enjeux sur lesquels ils travaillent. Les exposés de C. É. Bruzek sur le développement de câbles supraconducteurs et de S. Hébert sur les matériaux pour la thermoélectricité, ont tout particulièrement retenu l'attention.

2 - Les deux infinis était organisé par les divisions champs et particules et physique nucléaire. Trois sessions brossaient un panorama des recherches dans trois grandes thématiques de la physique des particules: la physique au LHC et à l'énergie du TeV, la physique des neutrinos et du mélange des quarks, et l'astroparticule ; les trois autres concernaient la physique de plus basse énergie, à savoir la physique hadronique, celle des noyaux exotiques et l'astrophysique nucléaire. L'assistance à ce minicolloque a été relativement importante (30 à 40 personnes à chaque session) et de très bonne qualité. Il faut cependant s'inquiéter de la faible participation de jeunes chercheurs.

3 - Le minicolloque Sources de rayonnement synchrotron et applications comportait quatre sessions, sur les accélérateurs, les sources et l'instrumentation, les lasers à électrons libres, les applications médicales, et la structure atomique et dynamique de la matière. Les propriétés remarquables du rayonnement synchrotron en termes de cohérence et d'accordabilité en énergie donnent lieu à des applications très prometteuses pour l'étude de la structure de nanostructures semi-conductrices (H. Renevier), pour imager sans lentille les propriétés structurales ou magnétiques (G. Beutier), ou pour coupler imagerie chimique et structurale aux échelles nanométriques (M.-I. Richard).

4 - Biophysique et matière molle était consacré tout particulièrement au thème du mouvement dans le monde vivant, depuis l'échelle microscopique (moteurs

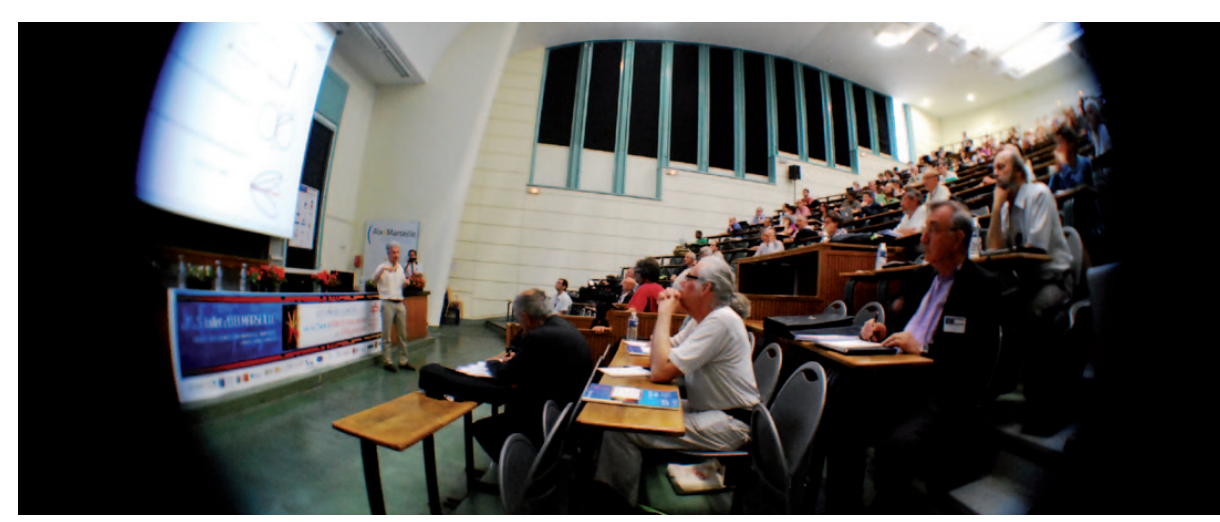

Conférence de François Amiranoff

moléculaires dans les cellules) jusqu'aux échelles macroscopiques du mouvement chez l'homme. Quatre sessions, quatre conférences invitées, seize communications orales et une dizaine d'affiches ont présenté les aspects les plus dynamiques de cette recherche à l'interface de la physique et de la biologie, où la communauté des physiciens français est particulièrement active et reconnue internationalement.

5 - Le minicolloque Physique théorique a rencontré un franc succès, avec une session Dynamique quantique : des mathématiques à la physique et aux questions d'interprétation faisant intervenir un mathématicien (F. Faure) et deux physiciens (R. Balian et D. Delande), et une session Physique et information : des trous noirs aux calculateurs quantiques, où sont intervenus un physicien, V. Balasubramanian (spécialiste à la fois de théorie des cordes et de neurosciences !) et un mathématicien, J. Slingerland. Une dernière session, organisée en commun avec la division de matière condensée, portait sur les applications à la Structure électronique de la matière condensée et approches ab initio.

6 - Dans le minicolloque Lasers intenses ont été traités pratiquement tous les aspects de l'interaction laser-matière, de la dynamique ultrarapide à la fusion par confinement inertiel, en passant par la physique à ultrahaute intensité. La biologie (A. Bonvalet), la physique attoseconde (V. Blanchet), l'optique relativiste (F. Quéré), l'accélération d'électrons (C. Thaury), la chimie (R. Cireasa), la physique du solide (P. Gilliot), les applications des lasers intenses (M. Sentis) et la fusion par confinement inertiel (Ph. Balcou) ont été abordés. À côté de ces exposés invités, une dizaine d'interventions ont été présentées, ainsi qu'une vingtaine de posters. L'affluence était au rendez-vous!
7 - Le minicolloque Plasmas s'est déroulé en deux sessions, l'une organisée conjointement avec le minicolloque Énergie, et l'autre dédiée aux Plasmas magnétisés. Les présentations ont porté sur les derniers développements dans les installations de fusion par confinement magnétique et inertiel et les potentialités de ces moyens expérimentaux, en soulignant les points d'achoppement où la recherche doit apporter des solutions. Un large panorama des plasmas magnétisés a été également présenté, allant des sources d'ions aux instabilités magnéto-hydrodynamiques, en passant par la propulsion spatiale.

8 - Futurs instruments de la physique subatomique était organisé conjointement par les divisions champs et particules, physique nucléaire et accélérateurs.

9 - Astrophysique de laboratoire aux basses énergies avait l'intention de mettre en avant l'apport essentiel des expériences de laboratoire à toute modélisation d'objet ou processus astrophysique. Une première session autour des données physico-chimiques a rassemblé des orateurs d'horizons divers, de la mesure des paramètres à la constitution de bases, et les échanges ont été particulièrement enrichissants. Dans la deuxième session sur les processus hydrodynamiques, les exposés ont montré, par leur diversité, combien il restait à apprendre dans la modélisation de ces phénomènes complexes, et combien l'expérience était indispensable.

10 - Refroidissement laser : des atomes à la matière a regroupé une cinquantaine de personnes. Les thèmes abordés furent le contrôle et l'utilisation du mouvement des atomes, mais aussi les avancées liées à d'autres systèmes (ions, molécules ou solides). 


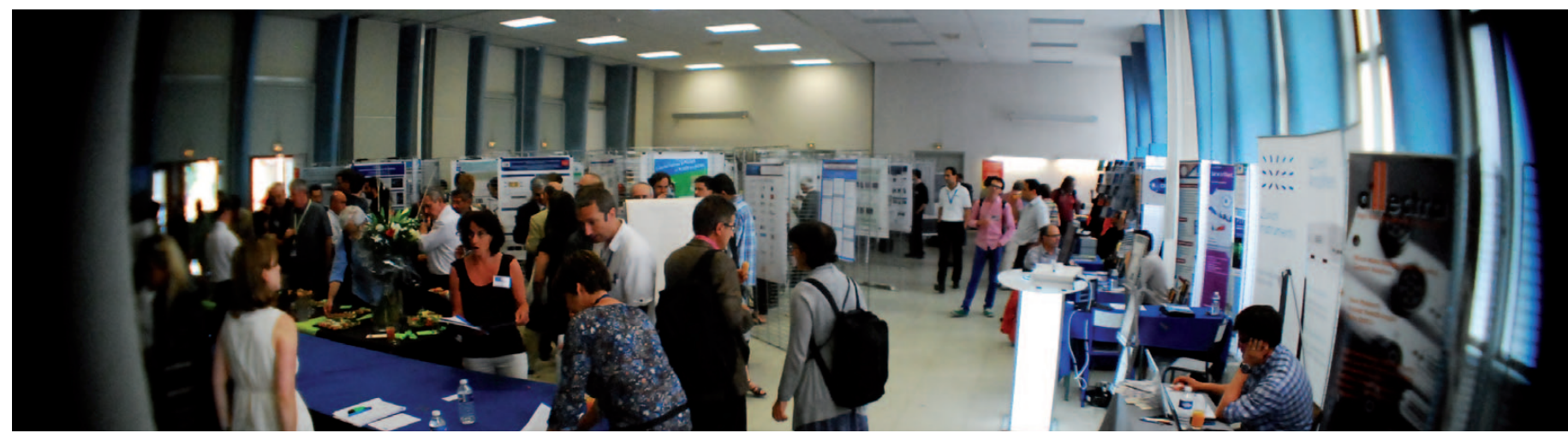

Séance poster auprès du stand des entreprises

Cette présentation, située volontairement hors des sentiers battus de la thématique habituelle, a ainsi permis de faire le point sur l'ouverture possible du domaine des atomes froids.

11 - Matériaux à corrélations électroniques fortes fut décliné en deux sessions. La première, intitulée Modes collectifs, a débuté avec une introduction très claire d'Henri Godfrin sur l'effet de l'interaction coulombienne appliquée aux modes collectifs dans l'hélium 3 liquide, suivie d'un impressionnant exposé d'Ariel Haziot sur la plasticité de l'hélium 4 solide. Le deuxième exposé invité, donné par Mathieu Le Tacon, traitait des modes magnons et de leur relation à la supraconductivité dans les cuprates à haute température critique. L'après-midi a vu la tenue de la session Ordres en compétition, avec deux exposés invités d'orateurs venus d'Allemagne, Christoph Geibel et Alexandre Pourret. La qualité des exposés était excellente, la science passionnante, les discussions vives et enthousiastes, mais les organisateurs ont été quelque peu déçus par le faible nombre de participants en fin de journée.

12 - Les deux sessions d'Imagerie en nanoscience étaient axées respectivement sur la microscopie électronique et sur la microscopie en champ proche. La première session a connu un franc succès, avec l'intervention remarquable du Prix Ancel 2012, Odile Stéphan, qui a exposé l'apport de différentes spectroscopies à la microscopie électronique en transmission. Tous les exposés ont été grandement appréciés par les auditeurs, la salle est restée comble pendant toute la session. La deuxième session a débuté par une superbe conférence de Sylvie Rousset, consacrée aux propriétés électroniques des nanostructures, et s'est poursuivie avec des applications de la microscopie en champ proche au photovoltaique organique (L. Letertre) et à la biologie (S. Scheuring). Une présentation par R. Morin du problème générique de la phase en imagerie a clos ce minicolloque, qui a donné lieu à des débats très animés.

13 - Cosmologie, organisé par la division champs et particules avec la SF2A, proposait également deux sessions : Énergie noire et matière noire et Formation et évolution des galaxies.

\section{4 - M. Knoop, B. van Tiggelen et} A. Henri animaient Évolution de l'édition scientifique. Après les exposés invités portant sur l'Open Access (A. Mahé), les critères, coûts et enjeux de la publication d'un article scientifique (J.M. Quilbé), les archives ouvertes (C. Berthaud) et la collaboration entre sociétés savantes et éditeurs commerciaux (J. Daillant), une table ronde a porté sur L'avenir des publications en physique. Le compte rendu de la table ronde est accessible sur le site : www.sfpnet.fr/ index.php?page $=$ tpage $\&$ id $=18$.

15 - Le minicolloque Enseignement a regroupé une cinquantaine de personnes. Les présentations des quatre chercheures en didactique de la physique ont permis de faire le point sur ce qu'est la démarche d'investigation (DI) et comment elle peut contribuer à la construction du savoir. Un compte rendu sera publié dans Reflets de la physique.

16 - Femmes et physique a réuni une soixantaine de participants (voir l'article dans ce numéro, pp. 38-39).

17-Enfin, leminicolloque Communication et culture scientifique s'ouvrait par une présentation de la politique gouvernementale en termes de culture scientifique (C. Forestier) ; l'organisation de la diffusion de la culture scientifique se fera en interministériel et s'appuiera sur les régions. $\mathrm{Ce}$ discours a été critiqué dans la discussion qui a suivi, en particulier pour le fait que la ministre écoute plus les associations et les ONG que les sociétés savantes. Ont suivi un exposé de F. Bouquet sur les diverses actions menées par l'équipe autour de J. Bobroff dans les lycées de la région parisienne, et une présentation du réseau Culture Sciences de la région PACA.

Jean-François Joanny ${ }^{(1)}$ et Dave Lollman ${ }^{(2)}$

(1) Président du Comité scientifique (jean-francois.joanny@espci.fr)

(2) Président du Comité d’organisation (dave.Iollman@univ-amu.fr)

Remerciements

Le $22^{\mathrm{e}}$ Congrès général de la SFP - Marseille 2013 a bénéficié du soutien de: Aix-Marseille Université, Université de Toulon, École Centrale de Marseille, le MESR, le CNRS, le CEA, Marseille Ville, la Région PACA, le Conseil Général CG13, Triangle de la Physique, EDP Sciences, EPL (Europhysics Letters), Fondation PSL Research University (Paris Sciences \& Lettres), POPsud/Optitec, Institut Carnot STAR, le GDR C'nano PACA, A`MIDEX - Labex OCEVU, MGEN, CASDEN, la Mission pour la place des femmes au CNRS, la Fédération Dynamique des Systèmes Complexes.

Au nom de la Société Française de Physique, JeanFrançois Joanny et Dave Lollman remercient tous les partenaires, l'ensemble du Comité scientifique et du Comité d'organisation, et tous les bénévoles.

\section{Le site web} www.sfp2013.fr

Vous y trouverez :

- Le programme complet.

- Les vidéos des conférences plénières

- Un reportage et un clip du Congrès général

- Les photos prises pendant le congrès 